posterior surface of the bladder between the two uteri and the anterior surface of the rectum. Suprapubic vaginal examination of the myomatous uterus was performed. On further examination the other uterus was found to have another tube and ovary placed upon its outer side. The patient made a good recovery.

On examination the vagina was found to be divided into two portions by a septum, and at the end of each canal was a distinct and separate cervix.

Three Cases of Oancer of the Cervix Complicating Irabor and Advanced Pregnancy, the Patients Remaining Well Fleven, Eight and One-half, and Fight Years after High Amputation of the Cervix. - SPENCER (Journal of Obstetries and Gynecology of the British Empire, December, 1904) reports the following cases:

Case I. Multipara, aged thirty-three years, was delivered by forceps, of a living child, at full term through a cancerous cervix a year before she came under Spencer's observation. The os dilated slowly, the posterior lip of the cervix dilating well, but the anterior lip, which was cancerous, did not yield. The membranes were ruptured artificially and labor progressed slowly. When the head was low in the pelvis a living child was delivered by forceps by very gentle traction. The placenta was expressed and the patient made a good recovery.

About a year after this the patient was admitted to the hospital in a pregnant condition, stating that she had had a discharge of blood and pus the last eight.months. On examination the fundus reached three and one-half inches above the pubes. The anterior lip of the cervix was greatly enlarged by an ulcerating malignant growth. The posterior lip was free. The cervix was removed by high amputation, the vagina was incised in front of the cervix, the bladder pushed up and a similar incision made behind, and the base of the broad ligament was tied with thick catgut ligatures, and the part between the cervix and the ligatures divided with scissors. The tissue was freed higher. up and the uterine cavity was opened anteriorly. The cervix was cut off and all bleeding stopped with a Paquelin cautery. The peritoneum was not opened. The vagina was packed with iodoform gauze and afterward boric acid douches and iodoform bougies were employed. She made a good recovery and left the hospital with her child in good condition. Subsequent pregnancy occurred, when the patient was delivered by Porro's operation, the ovaries and tubes being removed and the stump treated extraperitoneally. Eleven and a quarter years after the high amputation of the cervix the patient.was in good health. The abdominal and vaginal scars were sound and free from hernia.

Case II. was that of a multipara, who, on admission to the hospital, complained of discharge and bleeding, with pain. The patient had had eight children, but no miscarriages. On admission the patient was in fairly good condition, the uterus at six and a half months, and the child was living. The cervix was high up and much enlarged, with a malignant growth on the posterior lip. The patient was advised to have labor induced and the cervix removed, and finally consented. She was then delivered by induced labor and artificial dilatation. The child survived about half an hour. The patient recovered from labor without serious complications, and between two and three weeks afterward the cervix was removed by high amputation, the mother making a good 
recovery. More than eight and a half years after the removal of the cervix the patient was examined and found to be in good health. The scar was healthy, and there was no sign of recurrence.

Case III. was a multipara, who came to the hospital four and a half months after delivery, stating that she had had bleeding from the vagina daily since her confinement. On the left side of the posterior portion of the cervix there was a malignant growth. This was removed by high amputation, the patient making a good recovery. More than eight years after the operation the patient was well, the vaginal scars being normal and giving no evidence of malignant growth.

Spencer compares these results with those of the clinies at Leipzig and Berlin, giving the percentage of cures of 8.3 in operable cases, and in those too far advanced for radical cure treated by Cæesarean section, with a mortality of 80 per cent.

In his own experience he has had in hospital practice 6 cases of advanced pregnancy complicated with cancer of the cervix. Five of these were delivered through the vagina and operated upon during the puerperal period, and 1 of them was operated upon five months after parturition. All of these mothers recovered immediately from the operation. Two died seven months later, 1 twelve months later, and the other 3 remained well. One child died within half an hour, being premature; 1 died ten days afterward, while the remainder lived a number of months, sufficiently long to justify the method of treatment.

\section{GY NEOLOQY.}

\section{UNDHR THE CHARGE OF}

HENRY C. COE, M.D., OF NEW YORK.

ASSISTED BY

WILLIAM E. STUDDIFORD, M.D.

Radical Abdominal Operation for Cancer of the Uterus.-In a discussion of this subject before the Berlin Obstetrical and Gynecological Society, Bumm (Zentralblatt für Gynäkologie, 1905, No. 18) stated that he had had 75 cases, with 17 deaths, following operation; 56 patients had been under observation for over a year, of whom 23 were known to be well; 20 were free from recurrence at the end of two years. The speaker believed that a primary mortality of from 6 to 10 per cent. must imply an imperfect technique, since with the radical measures advocated by Mackenrodt a mortality of 20 per cent. was to be expected. With the extensive dissection necessary to remove all the diseased periuterine tissues it was impossible, he thought, to avoid a certain amount of septic infection, while there was always danger of injury to the ureters and bladder, not to speak of the risk of shock from such a prolonged operation. For these reasons he still gave the preference to the vaginal route in simple cases, in which the disease was confined to the uterus, and the organ was perfectly movable. 